\title{
Effets du taux de la lysine et de la méthionine de la ration sur les performances de croissance de la poule Barrée du Cameroun
}

\author{
Tamofo Alain Martial FOMEKONG, Jean Raphaël KANA*, Tadjong Ruben NGOUANA, \\ Kuetché Hervé MUBE and Alexis TEGUIA
}
Unité de Recherche en Production et Nutrition Animales (LAPRONAN), Département de Zootechnie, Faculté d'Agronomie et des Sciences Agricoles, Université de Dschang, Cameroun.
*Auteur correspondant; E-mail: kanajean@yahoo.fr

\section{RESUME}

Cette étude avait pour objectif de déterminer les besoins en lysine et en méthionine de la poule barrée du Cameroun. En démarrage (1 à 12 semaines), les rations expérimentales contenaient 1,2\% de lysine et $0,6 \%$ de méthionine $\left(D L_{1,2} M_{0,6}\right), 1,2 \%$ de lysine et $0,4 \%$ de méthionine $\left(D L_{1,2} M_{0,4}\right), 0,8 \%$ de lysine et $0,6 \%$ de méthionine $\left(D L_{0,8} M_{0,6}\right)$ et $0,8 \%$ de lysine et $0,4 \%$ de méthionine $\left(D L_{0,8} M_{0,4}\right)$. En phase croissance $(13$ à 20 semaines), les rations contenaient $0,8 \%$ de lysine et $0,3 \%$ de méthionine $\left(F L_{0,8} M_{0,3}\right), 0,8 \%$ de lysine et $0,5 \%$ de méthionine $\left(F L_{0,8} M_{0,5}\right), 0,7 \%$ de lysine et $0,3 \%$ de méthionine $\left(F L_{0,7} M_{0,3}\right)$ et $0,7 \%$ de lysine et $0,5 \%$ de méthionine $\left(F L_{0,7} M_{0,5}\right)$. En phase démarrage, les résultats ont révélé que l'indice de consommation (IC) est plus faible $(\mathrm{P}<0,05)$ avec la ration $D L_{1,2} M_{0,4}$ qui a été par ailleurs la ration la moins consommée. Les taux de la lysine et de la méthionine mis en évidence en phase de démarrage n'ont eu aucun effet significatif $(\mathrm{P}<0,05)$ sur le poids vif et le gain de poids à l'âge de 12 semaines. Par contre, à l'âge de 20 semaines, le poids vif a été significativement plus élevé avec $0,7 \%$ de lysine et $0,5 \%$ de méthionine $\left(F L_{0,7} M_{0,5}\right)$ chez la poule alors que chez le coq, c'est avec le même taux de lysine $(0,7 \%)$ et le taux de méthionine le plus faible $\left(F L_{0,7} M_{0,3}\right)$ que le poids vif le plus élevé a été enregistré. En conclusion, en phase démarrage, 1,2\% de lysine et $0,4 \%$ de méthionine sont nécessaires pour un meilleur IC alors qu'en phase croissance, 0,3 et $0,5 \%$ de méthionine permettent respectivement avec un taux constant de lysine $(0,7 \%)$ d'obtenir les meilleurs taux de croissance chez le coq et la poule Barrée camerounaise.

(C) 2018 International Formulae Group. All rights reserved.

Mots clés: Acide aminé, lysine, méthionine, performance de croissance, poule villageoise.

\section{Effects of ration content in lysine and methionine on the Cameroonian local barred chickens growth performances}

\begin{abstract}
This study was designed to determine the lysine and methionine needs of Cameroonian local barred chickens. At the starter phase ( 1 to 12 weeks), the experimental rations consisted of $\mathrm{D} L_{1.2} M_{0.6}(1.2 \%$ lysine and $0.6 \%$ methionine), $D L_{1.2} M_{0.4}\left(1.2 \%\right.$ lysine and $0.4 \%$ methionine), $D L_{0.8} M_{0.6}(0.8 \%$ lysine and $0.6 \%$ methionine $)$ and $D L_{0.8} M_{0.4}$ ( $0.8 \%$ lysine and $0.4 \%$ methionine). At the grower phase (13 to 20 weeks), the experimental rations consisted of $F L_{0.8} M_{0.3}(0.8 \%$ lysine and $0.3 \%$ methionine $), F L_{0.8} M_{0.5}(0.8 \%$ lysine and $0.5 \%$
\end{abstract}


methionine), $F L_{0.7} M_{0.3}\left(0.7 \%\right.$ lysine and $0.3 \%$ methionine) and $F L_{0.7} M_{0.5}(0.7 \%$ lysine and $0.5 \%$ methionine). At the starter phase, the smallest feed conversion ratio was recorded with the less consumed ration $D L_{1.2} M_{0.4}$. The levels of amino acids used at the starter phase has no significant effect on live body weight (LBW) and weight gain (WG) at 12 weeks old. At 20 weeks old, LBW was significantly higher with $0.7 \%$ lysine and $0.5 \%$ methionine in pullets while, in cock, the same level of lysine $(0.7 \%)$ and the lowest methionine level $(0.3 \%)$ induced the highest LBW. In conclusion, at the starter phase, $1.2 \%$ lysine and $0.4 \%$ methionine are necessary for a better growth while, 0.3 and $0.5 \%$ methionine respectively induced with $0.7 \%$ lysine the highest growth rate in cock and pullets barred chickens in the grower phase.

(C) 2018 International Formulae Group. All rights reserved.

Keywords: Amino acid, lysine, methionine, growth performance, local barred chickens.

\section{INTRODUCTION}

Une ration équilibrée joue un rôle important sur les performances de la poule villageoise à toutes les phases de la croissance (Lisnahan et al., 2017). Plusieurs études ont été conduites à l'effet de déterminer les besoins énergétiques (Magala et al., 2012; Alabi et al., 2013; Miah et al., 2014; 2016; Mube, 2016) et protéiques (Melesse et al., 2013; Banerjee et al., 2013; Liu et al., 2015; Miah et al., 2015) de ces poules en phase de croissance. Toutefois, la croissance des poules villageoises pourrait s'améliorer en ajustant les niveaux d'acides aminés essentiels dans la ration (Lisnahan et al., 2017). Il a été établi de plusieurs sources que chez la volaille, la méthionine et la lysine constituent les deux acides aminés limitant dans les rations à base de maïs et de tourteau de soja (Mingh et Ogle, 2005; Khan et al., 2011; Saki et al., 2011; Adbalqdadir et Arabi, 2014). Ils sont essentiels pour maximiser les performances de production et la qualité de la carcasse chez les poules en croissance (Gill, 2003; Sterling et al., 2006) et leur disponibilité dans la ration affecte la composition en acides aminés des protéines corporelles (Martin-Venegas et al., 2006; Conde-Aguilera et al., 2011).

Plusieurs études relatives aux acides aminés essentiels ont porté essentiellement sur les souches commerciales (Plumstead et al., 2007; Dozier et al., 2008; Kiess et al., 2013) et très peu de travaux se sont appesantis sur les besoins en acides aminés des poules villageoises à travers le monde. Pourtant, bien qu'elles affichent une croissance globalement lente par rapport aux souches commerciales, les poules villageoises font preuve d'une supériorité d'adaptation aux conditions environnementales locales et ont une qualité de viande meilleure qui cadre avec les exigences des marchés et les préférences des consommateurs (Yuan et al., 2015). Trisiwi et al. (2005) ont rapporté que la baisse du niveau de protéine de la ration de 18 à $16 \%$ associée à une supplémentation en méthionine et en lysine maintien les performances de croissance chez la poule villageoise Kampung. Par ailleurs, l'addition de $0,86 \%$ de lysine dans l'aliment servi aux poules villageoise âgés de 12 semaines améliore la croissance et l'efficacité alimentaire (Resnawati, 1998). Nasr et Kheiri (2011) ont préconisé l'augmentation des niveaux d'acides aminés limitant pour améliorer le gain de poids, la consommation alimentaire et l'indice de consommation chez la poule locale chinoise. A ce jour, aucune donnée relative aux besoins en acides aminés essentiels de la poule villageoise camerounaise n'est disponible dans la littérature. Ainsi, l'objectif de la présente étude est de déterminer les niveaux de lysine et de méthionine nécessaire pour optimiser les performances de croissance de la poule Barrée du Cameroun. 


\section{MATERIEL ET METHODES}

\section{Zone d'étude}

La présente étude a été menée dans l'Unité de Recherche en Aviculture de la Ferme d'Application et de Recherche (FAR) de la Faculté d'Agronomie et de Sciences Agricoles (FASA) de l'Université de Dschang (UDs). Dschang, ville montagneuse, est située à $1^{\prime}$ Ouest du Cameroun entre $5^{\circ} 26^{\prime}$ de latitude Nord, $10^{\circ} 26^{\prime}$ de longitude Est et à une altitude de $1420 \mathrm{~m}$ et une pluviométrie moyenne de $2000 \mathrm{~mm}$ par an.

\section{Matériel animal}

Au total, 240 poussins d'un jour non sexés de plumage identique (coucou) issus de l'incubation artificielle des œufs fertilisés produits à la Ferme d'Application et Recherche de l'Université de Dschang où s'est déroulée l'étude, ont été pesés dès le $1^{\mathrm{er}}$ jour et ont affiché un poids moyen de $31 \mathrm{~g}$. Lesdits poussins ont été répartis de manière aléatoire en quatre groupes, chacun étant constitué de quatre répétitions comptant chacune 15 sujets.

En phase finition, 224 sujets coucou sexés âgés de 12 semaines ont été répartis entre les différentes unités expérimentales suivant un dispositif factoriel $4 \times 2$ soit quatre traitements et 2 sexes (mâle et femelle).

Les poussins ont été vaccinés contre la maladie de Newcastle et la Bronchite infectieuse le $7^{\text {ème }}$ jour avec un rappel le $23^{\text {ème }}$ jour et contre la maladie de Gomboro le $10^{\text {ème }}$ jour. Des vitamines $\left(\right.$ Amintotal $\left.^{\circledR}\right)$ et un anticoccidien $\left(V^{2} a \operatorname{tacox}^{\circledR}\right.$ ) ont été administrés dans l'eau de boisson trois jours de suite toutes les deux semaines pendant la phase démarrage (1 à 12 semaines). L'anticoccidien $\left(\right.$ Vetacox $\left.^{\circledR}\right)$ et un déparasitant interne $\left(\right.$ Levalap $^{\circledR}$ ) ont été administrés toutes les trois semaines pendant la phase croissance (13 à 20 semaines).

\section{Logement}

Les poussins ont été élevés sur litière de copeaux de bois à une densité de 8 animaux $/ \mathrm{m}^{2}$ pendant la phase démarrage $(1$ à 12 semaines) et à une densité de $5 / \mathrm{m}^{2}$ dans les cages en bambou sur pilotis pendant la phase croissance (13 à 20 semaines). Au cours des deux phases (démarrage et finition), les sujets ont été nourris et abreuvés ad libitum.

\section{Rations expérimentales}

En phase démarrage, les rations expérimentales contenaient $1,2 \%$ de lysine et $0,6 \%$ de méthionine $\left(D L_{1,2} M_{0,6}\right), 1,2 \%$ de lysine et $0,4 \%$ de méthionine $\left(D L_{1,2} M_{0,4}\right)$, $0,8 \%$ de lysine et $0,6 \%$ de méthionine $\left(D L_{0,8} M_{0,6}\right)$, et $0,8 \%$ de lysine et $0,4 \%$ de méthionine $\left(D L_{0,8} M_{0,4}\right)$. En phase croissance, les rations contenaient $0,8 \%$ de lysine et $0,3 \%$ de méthionine $\left(F L_{0,8} M_{0,3}\right), 0,8 \%$ de lysine et $0,5 \%$ de méthionine $\left(F L_{0,8} M_{0,5}\right), 0,7 \%$ de lysine et $0,3 \%$ de méthionine $\left(F L_{0,7} M_{0,3}\right)$ et $0,7 \%$ de lysine et $0,5 \%$ de méthionine $\left(F L_{0,7} M_{0,5}\right)$. Les quatre rations (traitements) étaient comparables du point de vue de leur teneur en protéines brutes, en énergie métabolisable et en d'autres nutriments en phase démarrage (Tableau 1) et en phase croissance (Tableau 2).

\section{Collecte des données et paramètres étudiés}

Les valeurs moyennes de la consommation alimentaire et du poids vif ont été enregistrées de manière hebdomadaire. Le gain de poids hebdomadaire (GMH) a été obtenu en faisant la différence entre deux poids hebdomadaires consécutifs. L'indice de consommation (IC) a été évalué en faisant le rapport entre la quantité d'aliment consommée pendant la semaine sur le gain de poids hebdomadaire de la même semaine.

A l'âge de 20 semaines, 3 sujets par unité expérimentale soient 12 poules par traitement ont été soumis à une diète de 24 
heures, puis pesés, saignés, échaudés, plumés et éviscérés tel que décrit par Kana et al. (2015). Le poids relatif de chaque organe (gésier, foie, cœur) a été calculé de même que le poids relatif de quelques parties (tête, ailes, cuisses, bréchet et pattes) par rapport au poids vif. La longueur de l'intestin a été mesurée de la loupe duodénale au cloaque à l'aide d'un mètre ruban et la densité de l'intestin (poids de l'intestin/longueur de l'intestin) a été calculée.

\section{Analyses statistiques}

Les données sur la consommation alimentaire, le poids vif, l'indice de consommation et les caractéristiques de la carcasse ont été soumis à l'analyse de la variance (ANOVA) suivant un dispositif complètement randomisé. Le logiciel Statistical Package for Social Sciences (SPSS 12.0) a été utilisé pour les analyses. Lorsqu'ils existaient des différences entre les moyennes, le test de Duncan a été appliqué pour les séparer au seuil de signification de 5\%.

Tableau 1: Composition et valeur nutritive des rations expérimentales en phase démarrage.

\begin{tabular}{|c|c|c|c|c|}
\hline \multirow{2}{*}{ Ingrédients } & \multicolumn{4}{|c|}{ Rations expérimentales } \\
\hline & $D L_{1,2} M_{0,6}$ & $D L_{1,2} M_{0,4}$ & $D L_{0,8} M_{0,6}$ & $D L_{0,8} M_{0,4}$ \\
\hline Maïs & 59,22 & 59,22 & 57,9 & 56,9 \\
\hline Son de blé & 20,03 & 20,13 & 20,2 & 20 \\
\hline Tourteau de coton & 0,8 & 0,8 & 0 & 0,5 \\
\hline Tourteau soja & 8,7 & 8,7 & 1,7 & 1,7 \\
\hline Tourteau d'arachide & 0 & 0 & 10,9 & 10,9 \\
\hline Farine de poisson & 5,1 & 5,1 & 3,125 & 3,105 \\
\hline Farine d'os & 0 & 0 & 0 & 0,2 \\
\hline Coquillage & 0,75 & 0,85 & 0,945 & 0,965 \\
\hline CMAV 5\% ${ }^{(1)}$ & 5 & 5 & 5 & 5 \\
\hline Huile & 0 & 0 & 0,2 & 0,5 \\
\hline Lysine & 0,2 & 0,2 & 0 & 0 \\
\hline Méthionine & 0,2 & 0 & 0,03 & 0,23 \\
\hline Total & 100 & 100 & 100 & 100 \\
\hline \multicolumn{5}{|l|}{ Composition chimique calculée } \\
\hline Protéine brute (\%) & 18,00 & 18,02 & 18,00 & 18,07 \\
\hline Energie Métabolisable (kcal/kg) & 2800,68 & 2802,18 & 2801,23 & 2803,76 \\
\hline Calcium (\%) & 1,08 & 1,11 & 1,02 & 1,08 \\
\hline Phosphore disponible (\%) & 0,58 & 0,58 & 0,54 & 0,57 \\
\hline Lysine (\%) & 1,20 & 1,20 & 0,80 & 0,80 \\
\hline Méthionine (\%) & 0,60 & 0,40 & 0,60 & 0,40 \\
\hline Energie/Protéines & 155,59 & 155,50 & 155,62 & 155,16 \\
\hline
\end{tabular}

CMAV 5\%: Protéine brute $=40 \%$, Lysine $=3,3 \%$, Méthionine $=2,40 \%$, Calcium $=8 \%$, Phosphore $=2,05 \%$, Energie métabolisable $=2078 \mathrm{kcal} / \mathrm{kg} . \boldsymbol{D} \boldsymbol{L}_{1,2} \boldsymbol{M}_{0,6}:$ ration contenant $1,2 \%$ de lysine et $0,6 \%$ de méthionine, $\boldsymbol{D} \boldsymbol{L}_{1,2} \boldsymbol{M}_{0,4}:$ ration contenant $1,2 \%$ de lysine et $0,4 \%$ de méthionine, $\boldsymbol{D} \boldsymbol{L}_{0,8} \boldsymbol{M}_{0,6}:$ ration contenant $0,8 \%$ de lysine et $0,6 \%$ de méthionine, $\boldsymbol{D} \boldsymbol{L}_{0,8} \boldsymbol{M}_{0,4}:$ ration contenant $0,8 \%$ de lysine et $0,4 \%$ de méthionine. 
Tableau 2 : Composition et valeur nutritive des rations expérimentales en phase croissance.

\begin{tabular}{|c|c|c|c|c|}
\hline \multirow{2}{*}{ Ingrédients } & \multicolumn{4}{|c|}{ Rations expérimentales } \\
\hline & $F L_{0,8} M_{0,3}$ & $F L_{0,8} M_{0,5}$ & $F L_{0,7} M_{0,3}$ & $F L_{0,7} M_{0,5}$ \\
\hline Maïs & 52 & 52 & 54,50 & 54,50 \\
\hline Manioc & 15,50 & 15,25 & 12,00 & 11,75 \\
\hline Son de blé & 0 & 0 & 1,22 & 1 \\
\hline Tourteau de coton & 8 & 8 & 7 & 7 \\
\hline Tourteau soja & 14 & 14 & 8 & 8 \\
\hline Tourteau d'arachide & 3 & 3 & 10 & 10 \\
\hline Farine d'os & 4 & 4 & 3,75 & 4 \\
\hline Prémix $0,5 \%{ }^{(1)}$ & 0,5 & 0,5 & 0,5 & 0,5 \\
\hline Huile & 3 & 3 & 3 & 3 \\
\hline Méthionine & 0 & 0,25 & 0,03 & 0,25 \\
\hline Total & 100 & 100 & 100 & 100 \\
\hline \multicolumn{5}{|l|}{ Composition chimique calculée } \\
\hline Protéine brute (\%) & 16,32 & 16,31 & 16,28 & 16,24 \\
\hline Energie Métabolisable (kcal/kg) & 3025,98 & 3018,48 & 3025,74 & 3015,01 \\
\hline Calcium $(\%)$ & 1,23 & 1,23 & 1,12 & 1,22 \\
\hline Phosphore disponible (\%) & 0,71 & 0,72 & 0,71 & 0,74 \\
\hline Lysine (\%) & 0,86 & 0,86 & 0,78 & 0,78 \\
\hline Méthionine (\%) & 0,30 & 0,54 & 0,32 & 0,54 \\
\hline Energie/Protéines & 185,40 & 185,02 & 185,81 & 185,60 \\
\hline
\end{tabular}

${ }^{1}$ Prémix 0,5\%: lysine : $78000 \mathrm{mg} / \mathrm{kg}$, méthionine: $200000 \mathrm{mg} / \mathrm{kg}$, sulfate ferreux (monohydrate de fer): $10000 \mathrm{mg}$, oxyde de zinc $=10000 \mathrm{mg}$, sélénite de sodium $=20 \mathrm{mg}$, vitA : $3000000 \mathrm{UI}$, vitD $\mathrm{D}_{3}: 600000 \mathrm{UI}$, vitE 3000mg, vitB $_{1}: 320 \mathrm{mg}$, vitB $3 / \mathrm{Ac}$ panth: $2000 \mathrm{mg}$, vitB6: $400 \mathrm{mg}$, vitB12: $7 \mathrm{mg}$, Biotine: $10 \mathrm{mg}$, Acide folique: $150 \mathrm{mg}$, vitPP/Ac nicot/niacine: $6200 \mathrm{mg}$, choline chloride: $100000 \mathrm{mg}$. $\boldsymbol{F} \boldsymbol{L}_{0,8} \boldsymbol{M}_{0,3}:$ ration contenant $0,8 \%$ de lysine et $0,3 \%$ de méthionine, $\boldsymbol{F} \boldsymbol{L}_{0,8} \boldsymbol{M}_{0,5}:$ ration contenant $0,8 \%$ de lysine et $0,5 \%$ de méthionine, $\boldsymbol{F} \boldsymbol{L}_{0,7} \boldsymbol{M}_{0,3}$ : ration contenant $0,7 \%$ de lysine et $0,3 \%$ de méthionine, $\boldsymbol{F} \boldsymbol{L}_{0,7} \boldsymbol{M}_{0,5}$ : ration contenant $0,7 \%$ de lysine et $0,5 \%$ de méthionine.

\section{RESULTATS}

\section{Performance de croissance en phase démarrage}

Le Tableau 3 résume les performances pondérales et les données sur la consommation alimentaire des poules Barrées soumises à différents taux de lysine et de méthionine en phase démarrage. La consommation alimentaire la plus élevée $(\mathrm{P}<0,05)$ a été enregistrée avec la ration $D L_{0,8} M_{0,4}$ contenant les plus faibles teneurs en lysine et en méthionine $(0,8$ de lysine et 0,4 de méthionine) qui a, par ailleurs, été comparable à la ration $D L_{1,2} M_{0,6}(1,2$ de lysine et 0,6 de méthionine). Par contre, l'indice de consommation a été plus faible $(\mathrm{P}<0,05)$ avec la ration $D L_{1,2} M_{0,4}(1,2$ de lysine et 0,4 de méthionine) qui a été la ration la moins consommée de toutes les rations étudiées.

Les taux de la lysine et de la méthionine mis en évidence en phase de démarrage n'ont pas significativement $(\mathrm{P}<0,05)$ affecté le poids vif et le gain de poids à l'âge de 12 semaines. L'évolution du poids vif moyen des sujets en fonction des différentes rations (Figure 1) montre à 
l'analyse une allure et un profil identiques dans tous les groupes avec un léger avantage avec les taux les plus élevés de lysine et de méthionine $\left(D L_{1,2} M_{0,6}\right)$.

\section{Performance de croissance en phase croissance}

Les effets du taux de la lysine et de la méthionine de la ration et du genre sur la consommation alimentaire, le poids vif, le gain de poids, l'indice de consommation et le gain de poids de la poule Barrée en phase de croissance sont présentés dans le Tableau 4. De manière générale, il ressort de ce tableau qu'à traitement égal le sexe n'a pas d'effet significatif $(\mathrm{P}>0,05)$ sur tous les paramètres de croissances entre l'âge de 13 et 20 semaines d'âge. Indépendamment du sexe, aucune différence significative $(\mathrm{P}>0,05) \quad$ n'a été observée entre les différents traitements pour l'indice de consommation. Par contre, la consommation alimentaire des femelles a été plus élevée $(\mathrm{P}<0,05)$ avec les rations contenant le plus grand taux de méthionine $\left(F L_{0,8} M_{0,5}\right.$ et $\left.F L_{0,7} M_{0,5}\right)$ quel que soit le taux de lysine. Chez la poule, le poids vif a été significativement plus élevé avec $0,7 \%$ de lysine et $0,5 \%$ de méthionine $\left(F L_{0,7} M_{0,5}\right)$ alors que chez le coq, le poids vif le plus élevé a été enregistré avec le même taux de lysine $(0,7 \%)$ et le taux de méthionine $(0,3 \%)$ le plus faible $\left(F L_{0,7} M_{0,3}\right)$.

Indépendamment du sexe, les poids vifs les plus élevés $(\mathrm{P}<0,05)$ ont été enregistrés avec le taux de lysine le plus élevé $\left(F L_{0,8} M_{0,3}\right.$ et $\left.F L_{0,8} M_{0,5}\right)$ tandis que les plus faibles $(\mathrm{P}<0,05)$ ont été obtenues avec le plus faible taux de lysine $\left(F L_{0,7} M_{0,5}\right.$ et $\left.F L_{0,7} M_{0,3}\right)$ quel que soit le taux de méthionine. Par contre, chez le coq et la poule pris séparément, c'est avec le taux de lysine le plus faible $(0,7 \%)$ que les meilleures performances pondérales ont été enregistrées.
L'évolution du poids vif moyen des sujets en phase croissance en fonction des différents traitements et du sexe (Figure 2) montre, à l'analyse, une allure et un profil identique dans tous les groupes. Toutefois, sur toute la période de l'étude, la courbe de poids des poulettes soumises à $0,7 \%$ de lysine et $0,3 \%$ de méthionine $\left(F L_{0,7} M_{0,3}\right)$ est au dessus de celle de tous les autres groupes. Par contre, chez le coq, c'est avec le taux de méthionine le plus élevé $(0,5 \%)$ et le même taux de lysine $(0,7 \%)$ que la meilleure performance pondérale a été enregistrée.

\section{Développement des organes de digestion}

L'effet du taux de la lysine et de la méthionine de la ration sur le développement des organes de digestion est résumé dans le Tableau 5. Ces acides aminés, quel que soit le taux, ont significativement affecté $(\mathrm{P}<0,05)$ le développement des organes de digestion à l'exception de la longueur et de la densité de l'intestin. Chez les mâles, le poids relatif du gésier a été plus élevé $(\mathrm{P}<0,05)$ avec $0,8 \%$ de lysine et $0,5 \%$ de méthionine $\left(F L_{0,8} M_{0,5}\right)$. Indépendamment du sexe, l'intestin le plus lourd $(\mathrm{P}<0,05)$ a été enregistré avec la ration contenant $0,7 \%$ de lysine et $0,3 \%$ de méthionine $\left(F L_{0,7} M_{0,3}\right)$ par rapport aux rations contenant le taux de lysine le plus élevé $(0,8 \%)$.

Le Tableau 6 résume les caractéristiques de la carcasse de la poule Barrée camerounaise telles qu'affectées par le taux de la lysine et de la méthionine de la ration à l'âge de vingt semaines. Globalement, aucune différence significative n'a été enregistrée entre les différents traitements pour toutes les caractéristiques de la carcasse quel que soit le sexe de l'animal et le taux d'incorporation de ces acides aminés dans la ration. 
Tableau 3: Effets du taux de la lysine et de la méthionine sur les caractéristiques de croissance de la poule Barrée du Cameroun en phase démarrage.

\begin{tabular}{|c|c|c|c|c|c|c|}
\hline \multirow{2}{*}{$\begin{array}{l}\text { Caractéristiques } \\
\text { de la croissance }\end{array}$} & \multicolumn{4}{|l|}{ Rations } & \multirow{2}{*}{ ETM } & \multirow{2}{*}{$\mathbf{P}$} \\
\hline & $D L_{1,2} M_{0,6}$ & $D L_{1,2} M_{0,4}$ & $D L_{0,8} M_{0,6}$ & $D L_{0,8} M_{0,4}$ & & \\
\hline Consommation alimentaire $(\mathrm{g})$ & $3751,96^{b}$ & $2908,31^{\mathrm{a}}$ & $3571,17^{\mathrm{ab}}$ & $4824,35^{\mathrm{b}}$ & 829,91 & 0,002 \\
\hline Poids vif à 12 semaines (g) & 761,81 & 774,33 & 776,23 & 725,57 & 79,12 & 0,847 \\
\hline Gain de poids (g) & 711,57 & 733,52 & 731,51 & 683,51 & 77,36 & 0,847 \\
\hline Indice de consommation & $5,36^{\mathrm{ab}}$ & $4,15^{\mathrm{a}}$ & $4,85^{\mathrm{a}}$ & $7,08^{\mathrm{b}}$ & 1,44 & 0,019 \\
\hline
\end{tabular}

a, b: les moyennes portants les lettres identiques ne sont pas significativement différentes $(\mathrm{P}>0,05)$.

$\boldsymbol{D} \boldsymbol{L}_{1,2} \boldsymbol{M}_{0,6}$ : ration contenant $1,2 \%$ de lysine et $0,6 \%$ de méthionine, $\boldsymbol{D} \boldsymbol{L}_{1,2} \boldsymbol{M}_{0,4}:$ ration contenant $1,2 \%$ de lysine et $0,4 \%$ de méthionine,

$\boldsymbol{D} \boldsymbol{L}_{0,8} \boldsymbol{M}_{0,6}:$ ration contenant $0,8 \%$ de lysine et $0,6 \%$ de méthionine, $\boldsymbol{D} \boldsymbol{L}_{0,8} \boldsymbol{M}_{0,4}$ : ration contenant $0,8 \%$ de lysine et $0,4 \%$ de méthionine

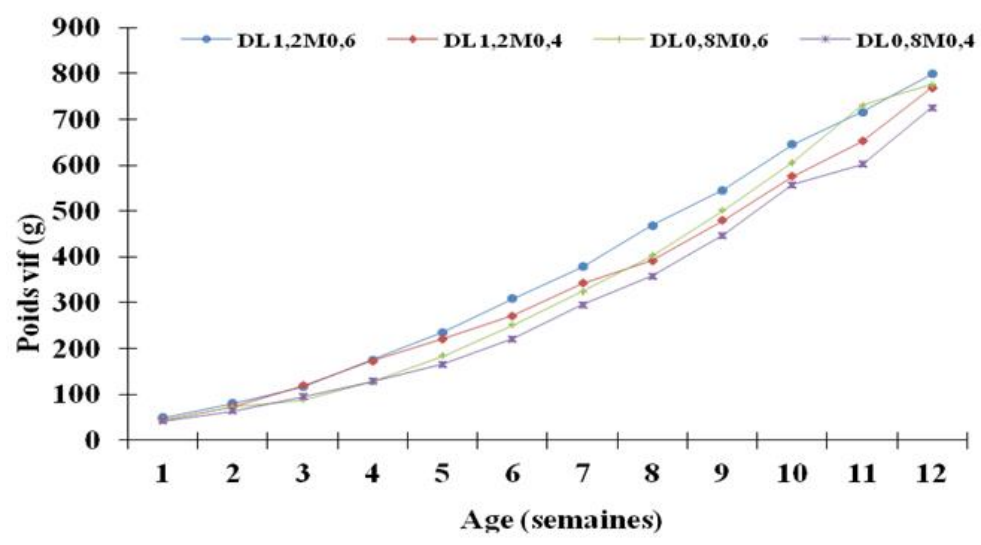

Figure 1: Evolution hebdomadaire du poids vif de la poule barrée camerounaise en fonction du taux de la lysine et de la méthionine en phase démarrage.

$\boldsymbol{D} \boldsymbol{L}_{1,2} \boldsymbol{M}_{0,6}:$ ration contenant $1,2 \%$ de lysine et $0,6 \%$ de méthionine, $\boldsymbol{D} \boldsymbol{L}_{1,2} \boldsymbol{M}_{0,4}:$ ration contenant $1,2 \%$ de lysine et $0,4 \%$ de méthionine, $\boldsymbol{D} \boldsymbol{L}_{0,8} \boldsymbol{M}_{0,6}:$ ration contenant $0,8 \%$ de lysine et 0,6\% de méthionine, $\boldsymbol{D} \boldsymbol{L}_{0,8} \boldsymbol{M}_{0,4}:$ ration contenant $0,8 \%$ de lysine et $0,4 \%$ de méthionine.
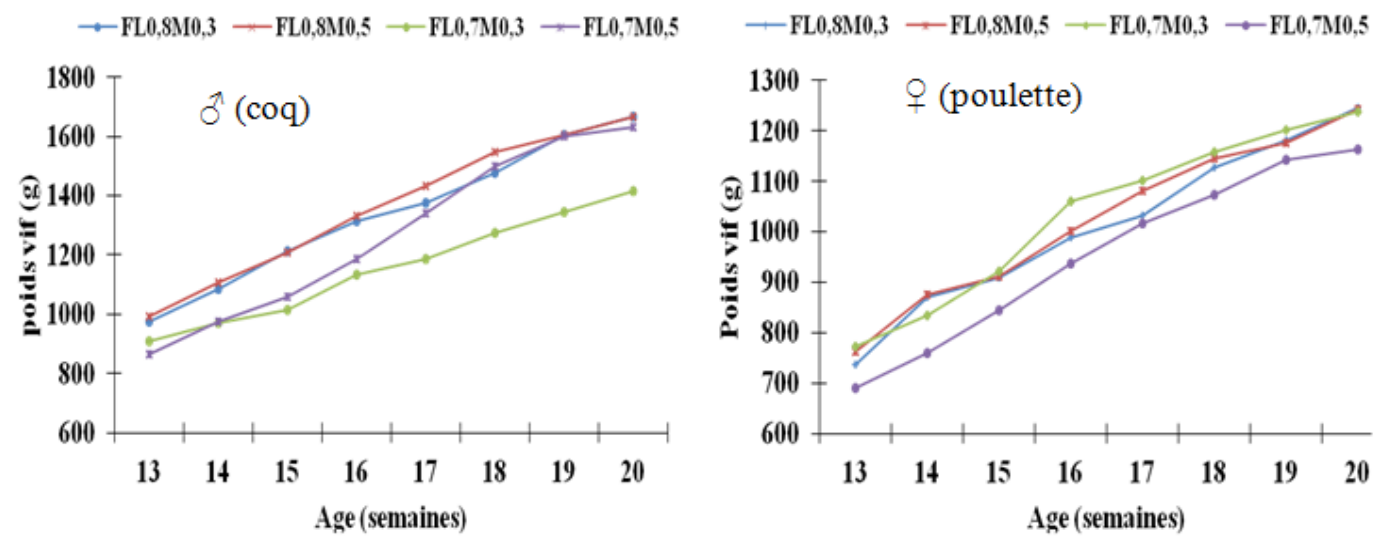

Figure 2: Evolution hebdomadaire du poids vif de la poulette et du coq en fonction du taux de lysine et de méthionine en phase croissance.

$\boldsymbol{F} \boldsymbol{L}_{0,8} \boldsymbol{M}_{0,3}$ : ration contenant $0,8 \%$ de lysine et $0,3 \%$ de méthionine, $\boldsymbol{F} \boldsymbol{L}_{0,8} \boldsymbol{M}_{0,5}$ : ration contenant $0,8 \%$ de lysine et $0,5 \%$ de méthionine, $\boldsymbol{F} \boldsymbol{L}_{0,7} \boldsymbol{M}_{0,3}:$ ration contenant $0,7 \%$ de lysine et 0,3\% de méthionine, $\boldsymbol{F} \boldsymbol{L}_{0,7} \boldsymbol{M}_{0,5}:$ ration contenant $0,7 \%$ de lysine et $0,5 \%$ de méthionine. 
Tableau 4 : Effets du taux de la lysine et de la méthionine sur les performances de croissance de la poule Barrée entre 13 et 20 semaines d'âge.

\begin{tabular}{|c|c|c|c|c|c|c|}
\hline \multirow{2}{*}{ Sexes } & \multicolumn{4}{|l|}{ Rations } & \multirow{2}{*}{ SEM } & \multirow{2}{*}{$\mathbf{P}$} \\
\hline & $F L_{0,8} M_{0,3}$ & $F L_{0,8} M_{0,5}$ & $F L_{0,7} M_{0,3}$ & $F L_{0,7} M_{0,5}$ & & \\
\hline \multicolumn{7}{|c|}{ Consommation alimentaire (g) } \\
\hline q & $4307,14^{\mathrm{a}}$ & $5813,69^{\mathrm{ab}}$ & $4575,51^{\mathrm{a}}$ & $6743,02^{b}$ & 1275,60 & 0,019 \\
\hline$\hat{0}$ & 5483,21 & 5626,64 & 5695,91 & 5920,88 & 1621,48 & 0,988 \\
\hline$\widehat{o}$ o우 & 5851,08 & 4938,53 & 4910,94 & 6093,84 & 1267,34 & 0,460 \\
\hline $\mathrm{P}$ & 0,305 & 0,292 & 0,144 & 0,873 & & \\
\hline \multicolumn{7}{|c|}{ Poids vif (g) } \\
\hline q & $1218,95^{\mathrm{a}}$ & $1458,11^{\text {ab }}$ & $1480,65^{\mathrm{ab}}$ & $1656,78^{b}$ & 255,36 & 0,042 \\
\hline$\hat{0}$ & $1245,06^{\mathrm{a}}$ & $1238,34^{\mathrm{a}}$ & $1668,01^{\mathrm{b}}$ & $1526,95^{\mathrm{b}}$ & 120,37 & 0,000 \\
\hline jot & $1456,79^{\mathrm{b}}$ & $1470,68^{\mathrm{b}}$ & $1302,95^{\mathrm{a}}$ & $1397,09^{\mathrm{ab}}$ & 94,73 & 0,028 \\
\hline $\mathrm{P}$ & 0,901 & 0,073 & 0,129 & 0,356 & & \\
\hline \multicolumn{7}{|c|}{ Gain de poids (g) } \\
\hline q & $441,37^{\mathrm{a}}$ & $614,95^{\mathrm{ab}}$ & $501,21^{\mathrm{a}}$ & $826,52^{b}$ & 207,75 & 0,057 \\
\hline$\hat{\sigma}$ & 554,09 & 630,46 & 674,30 & 654,88 & 172,19 & 0,789 \\
\hline o우 & 628,76 & 617,05 & 462,93 & 648,50 & 128,24 & 0,142 \\
\hline $\mathrm{P}$ & 0,265 & 0,402 & 0,100 & 0,524 & & \\
\hline \multicolumn{7}{|c|}{ Indice de consommation } \\
\hline$q$ & 9.92 & 9.54 & 10.69 & 8.28 & 2,61 & 0,730 \\
\hline$\hat{0}$ & 9,71 & 10,05 & 8,67 & 9,27 & 2,94 & 0,913 \\
\hline$\widehat{\jmath} 0+$ & 9,26 & 8,13 & 11,06 & 9,60 & 2,17 & 0,317 \\
\hline $\mathrm{P}$ & 0,151 & 0,251 & 0,057 & 0,386 & & \\
\hline
\end{tabular}

a, b: les moyennes portant la même lettre dans la même ligne ne sont pas significativement différentes $(\mathrm{P}>0,05)$

$\boldsymbol{F} \boldsymbol{L}_{0,8} \boldsymbol{M}_{0,3}$ : ration contenant $0,8 \%$ de lysine et $0,3 \%$ de méthionine, $\boldsymbol{F} \boldsymbol{L}_{0,8} \boldsymbol{M}_{0,5}:$ ration contenant $0,8 \%$ de lysine et $0,5 \%$ de méthionine, $\boldsymbol{F} \boldsymbol{L}_{0,7} \boldsymbol{M}_{0,3}:$ ration contenant $0,7 \%$ de lysine et $0,3 \%$ de méthionine, $\boldsymbol{F} \boldsymbol{L}_{0,7} \boldsymbol{M}_{0,5}:$ ration contenant $0,7 \%$ de lysine et $0,5 \%$ de méthionine

Tableau 5: Effets du taux de la lysine et de la méthionine de la ration sur le développement des organes de digestion de la poule Barrée camerounaise.

\begin{tabular}{|c|c|c|c|c|c|c|c|}
\hline \multirow[b]{2}{*}{ Organes } & \multirow[b]{2}{*}{ Sexe } & \multicolumn{4}{|l|}{ Rations } & \multirow[b]{2}{*}{ ETM } & \multirow[b]{2}{*}{$\mathbf{P}$} \\
\hline & & $F L_{0,8} M_{0,3}$ & $F L_{0,8} M_{0,5}$ & $F L_{0,7} M_{0,3}$ & $F L_{0,7} M_{0,5}$ & & \\
\hline \multirow{3}{*}{$\begin{array}{l}\text { Poids relatif } \\
\text { du gésier }(\%)\end{array}$} & $0^{\pi}$ & $1,87^{\mathrm{ab}}$ & $2,10^{b}$ & $1,69^{\mathrm{a}}$ & $1,73^{\mathrm{a}}$ & 0,25 & 0,030 \\
\hline & q & 1,92 & 1,76 & 2,06 & 1,83 & 0,35 & 0,846 \\
\hline & & 1,71 & 1,77 & 2,00 & 1,95 & 0,23 & 0,240 \\
\hline \multirow{3}{*}{$\begin{array}{l}\text { Poids de } \\
\text { l'intestin (g) }\end{array}$} & $\sigma^{\pi}$ & 2,92 & 3,95 & 2,81 & 3,22 & 0,86 & 0,148 \\
\hline & o & 3,30 & 2,68 & 3,20 & 2,80 & 0,77 & 0,756 \\
\hline & ऽ우 & $2,69^{\mathrm{a}}$ & $2,91^{\mathrm{a}}$ & $3,81^{\mathrm{b}}$ & $3,06^{\mathrm{ab}}$ & 0,66 & 0,005 \\
\hline \multirow{3}{*}{$\begin{array}{l}\text { Longueur de } \\
\text { l'intestin } \\
\text { (cm) }\end{array}$} & $\hat{\sigma}$ & 118,91 & 111,16 & 125,30 & 125,18 & 11,46 & 0,178 \\
\hline & q & 115,75 & 118,31 & 117,50 & 124,63 & 8,16 & 0,606 \\
\hline & ふ̄o & 122,38 & 126,56 & 123,33 & 126,43 & 9,43 & 0,923 \\
\hline Densité de & $\hat{\sigma}$ & 0,34 & 0,51 & 0,38 & 0,36 & 0,18 & 0,513 \\
\hline l'intestin & o & 0,37 & 0,33 & 0,38 & 0,38 & 0,06 & 0,698 \\
\hline$(\mathrm{g} / \mathrm{cm})$ & ito & 0,34 & 0,38 & 0,39 & 0,44 & 0,11 & 0,779 \\
\hline
\end{tabular}

a, b: les moyennes portant la même lettre sur la même colonne ne sont pas significativement différentes $(\mathrm{P}>0,05)$

$\boldsymbol{F L}_{0,8} \boldsymbol{M}_{0,3}:$ ration contenant $0,8 \%$ de lysine et $0,3 \%$ de méthionine, $\boldsymbol{F} \boldsymbol{L}_{0,8} \boldsymbol{M}_{0,5}:$ ration contenant $0,8 \%$ de lysine et $0,5 \%$ de méthionine, $\boldsymbol{F} \boldsymbol{L}_{0,7} \boldsymbol{M}_{0,3}$ : ration contenant 0,7\% de lysine et 0,3\% de méthionine, $\boldsymbol{F} \boldsymbol{L}_{0,7} \boldsymbol{M}_{0,5}$ : ration contenant $0,7 \%$ de lysine et $0,5 \%$ de méthionine 
Tableau 6 : Effets du taux de la lysine et de la méthionine sur les caractéristiques de la carcasse (\%) de la poule Barrée camerounaise.

\begin{tabular}{|c|c|c|c|c|c|c|c|}
\hline \multirow{2}{*}{$\begin{array}{l}\text { Caractéristiques } \\
\text { de la carcasse } \\
\text { (\% du poids vif) }\end{array}$} & \multirow{2}{*}{ Sexe } & \multicolumn{4}{|l|}{ Rations } & \multirow{2}{*}{ ETM } & \multirow{2}{*}{$\mathbf{P}$} \\
\hline & & $F L_{0,8} M_{0,3}$ & $F L_{0,8} M_{0,5}$ & $F L_{0,7} M_{0,3}$ & $F L_{0,7} M_{0,5}$ & & \\
\hline \multirow{3}{*}{$\begin{array}{l}\text { Rendement } \\
\text { carcasse (\%) }\end{array}$} & $\hat{0}$ & 61,67 & 68,54 & 67,86 & 67,19 & & \\
\hline & q & 67,13 & 66,52 & 64,08 & 76,41 & & \\
\hline & ôto & 64,03 & 68,34 & 67,43 & 73,99 & 8,61 & 0,470 \\
\hline \multirow{3}{*}{$\begin{array}{l}\text { Poids relatif du } \\
\text { Foie }(\%)\end{array}$} & $\hat{\sigma}$ & 1,82 & 1,72 & 1,57 & 1,70 & & \\
\hline & q & 1,64 & 1,84 & 1,81 & 1,66 & & \\
\hline & o우 & 1,79 & 1,54 & 1,78 & 1,67 & 0,16 & 0,151 \\
\hline \multirow{3}{*}{$\begin{array}{l}\text { Poids relatif du } \\
\text { Cœur (\%) }\end{array}$} & $0^{1}$ & 0,52 & 0,58 & 0,56 & 0,59 & & \\
\hline & o & 0,55 & 0,62 & 0,57 & 0,55 & & \\
\hline & $0^{1}+0$ & 0,56 & 0,57 & 0,57 & 0,56 & 0,04 & 0,979 \\
\hline \multirow{3}{*}{$\begin{array}{l}\text { Poids relative des } \\
\text { cuisses }(\%)\end{array}$} & $\hat{0}$ & 19,71 & 20,01 & 21,48 & 21,87 & & \\
\hline & q & 20,30 & 20,95 & 21,62 & 21,65 & & \\
\hline & o우 & 20,60 & 21,04 & 20,70 & 21,11 & 0,65 & 0,697 \\
\hline \multirow{3}{*}{$\begin{array}{l}\text { Poids relative de } \\
\text { la } \quad \text { graisse } \\
\text { abdominale }(\%)\end{array}$} & $\hat{\sigma}$ & 5,56 & 4,81 & 3,87 & 2,23 & & \\
\hline & q & 3,90 & 3,67 & 2,54 & 3,29 & & \\
\hline & o우 & 4,77 & 4,07 & 3,15 & 3,84 & 0,97 & 0,117 \\
\hline \multirow{3}{*}{$\begin{array}{l}\text { Poids relatif du } \\
\text { bréchet }(\%)\end{array}$} & $\hat{0}$ & 17,11 & 18,05 & 15,82 & 15,64 & & \\
\hline & 우 & 16,14 & 15,87 & 15,01 & 15,70 & & \\
\hline & o우 & 16,22 & 15,96 & 16,68 & 16,41 & 0,92 & 0,807 \\
\hline \multirow{3}{*}{$\begin{array}{l}\text { Poids relatif de la } \\
\text { tête }(\%)\end{array}$} & 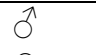 & 2,62 & 3,01 & 2,87 & 3,25 & & \\
\hline & q & 2,74 & 3,55 & 3,18 & 3,32 & & \\
\hline & ôto & 4,52 & 2,75 & 3,12 & 3,12 & 1,67 & 0,525 \\
\hline \multirow{3}{*}{$\begin{array}{l}\text { Poids relatif des } \\
\text { pattes }(\%)\end{array}$} & $\hat{0}$ & 2,64 & 2,95 & 3,21 & 3,49 & & \\
\hline & 우 & 3,01 & 3,87 & 3,50 & 3,35 & & \\
\hline & ôto & 2,88 & 3,07 & 3,39 & 3,42 & 0,42 & 0,204 \\
\hline
\end{tabular}

$\boldsymbol{F} \boldsymbol{L}_{0,8} \boldsymbol{M}_{0,3}$ : ration contenant $0,8 \%$ de lysine et 0,3\% de méthionine, $\boldsymbol{F L}_{0,8} \boldsymbol{M}_{0,5}$ : ration contenant 0,8\% de lysine et $0,5 \%$ de méthionine, $\boldsymbol{F} \boldsymbol{L}_{0,7} \boldsymbol{M}_{0,3}$ : ration contenant $0,7 \%$ de lysine et $0,3 \%$ de méthionine, $\boldsymbol{F} \boldsymbol{L}_{0,7} \boldsymbol{M}_{0,5}$ : ration contenant $0,7 \%$ de lysine et $0,5 \%$ de méthionine

\section{DISCUSSION}

En démarrage, la consommation alimentaire a été plus élevée avec les plus faibles teneurs en lysine et en méthionine $(0,8$ et 0,4 respectivement). Cette observation corrobore les rapports dressés par Stilborn et al. (1997) chez les poulets de chair et Lisnahan et al. (2017) chez la poule villageoise faisant état de ce que les poulets nourris par les faibles teneurs en acides aminés tendent à augmenter leur consommation alimentaire. Ceci serait le reflet de l'équilibre de la ration en acides aminés. A propos, Jackson et al. (1982) ont rapporté que le déséquilibre en acides aminés de la ration diminue la valeur biologique de celle-ci et, par conséquent, altère la consommation alimentaire. L'influence non significative des taux de lysine et de méthionine mis en évidence en phase de démarrage sur le poids vif à 12 semaines confirme les résultats de $\mathrm{Si}$ et al. (2004) qui ont, à cet effet, affirmé qu'en phase démarrage, l'augmentation de la densité en acides aminés de la ration n'altère pas le poids vif final de la poule.

En phase finition, la consommation la plus élevée a été enregistrée chez les poules 
soumises aux taux de méthionine les plus élevés. Un rapport similaire a été dressé par Lisnahan et al. (2017). Par ailleurs, Swennen et al. (2011) ont rapporté que lorsque les quantités optimales d'acides aminés sont présentes dans l'aliment, les poules augmentent la consommation alimentaire pour couvrir leur besoins. Si et al. (2004) ont renchérit que ce n'est pas seulement la protéine qui est importante pour couvrir les besoins nutritionnels mais la présence des quantités équilibrées en acides aminés est aussi requise. Indépendamment du sexe, les taux de lysine et de méthionine les plus élevés ont tendance à baisser bien que non significativement l'indice de consommation en phase croissance suggérant ainsi une meilleure utilisation alimentaire. $\mathrm{La}$ conséquence immédiate a été l'amélioration du poids vif des sujets consommant ces rations durant cette phase. Dans le même sens, Lisnahan et al. (2017) ont soutenu que le taux de croissance peut rapidement augmenter en ajoutant ces acides aminés dans la ration parce qu'ils sont immédiatement absorbés dans l'organisme et que, contrairement aux acides aminés consommés comme constituants de la protéine totale, les acides aminés individuels ne requiert pas de digestion préalable.

Les poids vifs les plus élevés ont été enregistrés avec le taux de lysine le plus élevé ( $0,8 \%$ de lysine) indépendamment de celui de la méthionine tandis que l'inverse a été observé chez les mâles pour ce qui est du gain de poids. Plusieurs autres travaux ont rapporté des résultats similaires (Onu et al., 2010; Lisnahan et al., 2017). L'amélioration observée sur le poids vif et le gain de poids des oiseaux soumis aux taux de lysine et de méthionine les plus élevés pourrait être dû à l'effet de la disponibilité de ces deux acides aminés limitant sur l'amélioration de la digestibilité et une meilleure absorption des nutriments (Onu et al., 2010; Lisnahan et al., 2017). Ceci corrobore les résultats de plusieurs autres travaux effectués en aviculture (Cafe et Waldroup, 2006; Domingues et al., 2012) montrant que les acides aminés essentiels que sont la lysine et la méthionine constituent les nutriments les plus importants chez les poules en croissance. Ces études ont aussi montré que le poids des poules est positivement affecté par la disponibilité et l'équilibre des acides aminés dans l'aliment qu'elles consomment. La synthèse protéique au niveau des ribosomes est hautement dépendante de la présence des acides aminés requis par les tissus; par conséquent, l'efficacité et le taux de synthèse protéique sont fortement influencés par l'équilibre et la suffisance des acides aminés donnant ainsi lieu à une augmentation du gain de poids (Onu et al., 2010).

Le résultat de la présente étude suggère que le taux d'inclusion de la lysine de 0,8\% satisfait les besoins de la poule Barrée en croissance et se trouverait dans les limites acceptables pour accélérer la synthèse protéique dans les tissus. Indépendamment du sexe des oiseaux, la baisse significative du poids vif avec les plus faibles taux des deux acides aminés suggère que ces taux sont en dessous des besoins de croissance de la poule barrée du Cameroun. Cette observation consolide les résultats d'Etuk et al. (2004) et Rezaei et al. (2004) et donne aussi de la crédibilité au fait que le taux et la valeur biologique de la protéine de la ration doivent être suffisamment élevés pour fournir les acides aminés suffisants nécessaire à la construction des protéines corporelles (Onu et al., 2010).

Aucune différence significative n'a été observée entre les traitements pour ce qui est des caractéristiques de la carcasse. Un rapport similaire a déjà été dressé par Onu et al. (2010). On a cependant noté une légère augmentation numérique du poids relatif du bréchet chez les coqs et les poules recevant le taux de lysine le plus élevé $(0,8 \%)$. Une observation similaire a été faite par Rezaei et al. (2004) et Onu et al. (2010). Ceci confirme qu'en règle générale, la lysine joue un rôle important dans la formation du tissu du bréchet, composante majeure de la carcasse (Si et al., 2004; Sterling et al., 2006; Abbas, 2015). 


\section{Conclusion}

Les résultats présentés dans cette étude indiquent que la lysine et la méthionine sont des acides aminés critiques chez la poule Barrée du Cameroun. Une méthode pour améliorer ses performances de croissance consisterait à ajouter à l'aliment ces acides aminés aux taux de 1,2 et $0,4 \%$ respectivement pour la lysine et la méthionine en phase démarrage, $0,7 \%$ de lysine quel que soit le sexe et 0,3 et $0,5 \%$ de méthionine chez le coq et la poule respectivement en phase croissance.

\section{CONFLIT D'INTERETS}

Les auteurs déclarent n'avoir aucun conflit d'intérêts.

\section{CONTRIBUTION DES AUTEURS}

FTAM, KJR et AT ont contribué à la conception et à la planification des expériences. FTAM, NTR et MKH ont collecté les données sur le terrain. NTR et MKH ont effectué les analyses statistiques des données et FTAM et KJR ont rédigé le manuscrit sur la supervision de AT.

\section{RÉFÉRENCES}

Abbas TE. 2015. Threonine-Lysine Ratio and its Effect on Broiler Performance. International Journal in Advanced Research in Biological Science, 2: 174179.

Adbalqdadir MO, Arabi SA. 2014. The Effects of Different Lysine and Methionine Levels on Broiler Chickens Performance. International Journal of Innovative Agriculture and Biological Resources, 2(4): 46-52.

Alabi OJ, Ng'ambi JW, Norris D. 2013. Dietary energy level for optimum productivity and carcass characteristics of indigenous Venda chickens raised in closed confinement. South Africa Journal of Animal Science, 43(1): 75-80. http://dx.doi.org/10.4314/sajas.v43i5

Banerjee SA, Melesse E, Dotamo K, Berihun, Beyan M. 2013. Effect of feeding different dietary protein levels with iso- caloric ration on nutrients intake and growth performances of dual Purpose Koekoeck chicken breeds. International Journal of Apply Poultry Research, 2(2): 27-32.

Cafe MB, Waldroup PW. 2006. Interactions Between Levels of Methionine and Lysine in Broiler Diets Changed at Typical Industry Intervals. International Journal of Poultry Science, 5: 10081015.

Conde-Aguilera JA, Aguinaga MA, Lara L, Aguilera JF, Nieto R. 2011. Carcass Traits and Organ Weights of 10-25-kg Body Weight Iberian Pigs Fed Diets with Different Protein-to-Energy Ratio. Animal Feed Science and Technology, 164: 116-124.

Domingues CHDP, Sgavioli S, Praes MFFM, Duarte KF, Castiblanco DMC. 2012. Lysine and Methionine+ Cystine for molting phase. Brazilian Journal of Poultry Science, 14: 159-232.

Dozier WA, Corzo A, Kidd MT, Schilling MW. 2008. Dietary Digestible Lysine Requirements of Male and Female Broilers from Forty-Nine to Sixty-Three Days of Age. Poultry Science, 87: 13851391.

Etuk EB, Esonu BO, Njoku C. 2004. The effect of Methionine Supplementation on the Performance of Finisher Broilers Fed Pigeon Pea (Cajanus cajan) Seed Meal Based Diets. Tropical Journal of Animal Science, 7(2): 37-42.

Gill C. 2003. Pig and Poultry: Value-Added' Ingredients or More Amino Acids? Feed International, 24: 27-29.

Jackson S, Summers JD, Leeson S. 1982. Effect of Dietary Protein and Energy on Broiler Performance and Production Cost. Poultry Science, 61: 2232-2240.

Kana JR, Hassan AD, Ngouana TR, Mube KH, Teguia A, Tefack Y, Zambou HR. 2015. Performances zootechniques du poulet de chair soumis à un régime à base de résidus de semoule de manioc supplémenté par la spiruline (Spirulina platensis). International Journal of 
Biological and Chemical Sciences, 9(5): 2607-2616.

Khan SA, Ujjan NU, Ahmed G, Rind MI, Fazlani SA, Faraz S, Ahmed S, Asif M. 2011. Effect of low Protein Diet Supplemented with or without AminoAcids on the Production of Broiler. African Journal of Biotechnology, 10(49): 10058-10065.

Kiess AS, Manangi MK, Cleveland BM, Wilson ME, Blemings KP. 2013 Effect of Dietary Lysine on Hepatic Lysine Catabolism in Broilers. Poultry Science, 92: 2705-2712.

Lisnahan CV, Wihandoyo, Zuprizal, Sri Harimurti. 2017. Growth Performance of Native Chickens in the Grower Phase Fed Methionine and LysineSupplemented Cafeteria Standard Feed. Pakistan Journal of Nutrition, 16(12): 940-944. doi=pjn.2017.940.944\&linkid=pdf

Liu SK, Niu ZY, Min YN, Wang ZP, Zhang J, He ZF, Li HL, Sun TT, Liu FZ. 2015. Effects of Dietary Crude Protein on the Growth Performance, Carcass Characteristics and Serum Biochemical indexes of Lueyang Black-boned Chickens from Seven to Twelve Weeks of Age. Brazilian Journal of Poultry Science, 17(1): 103-108.

Magala H, Kugonza DR, Kwizera H, Kyarisiima CC. 2012. Influence of Varying Dietary Energy and Protein on Growth and Carcass Characteristics of Ugandan Local Chickens. Journal of Animal Production Advanced, 2(7): 316324.

Martin-Venegas R, Geraert PA, Ferrer R. 2006. Conversion of the Methionine Hydroxy analogue DL-2hydroxy-(4methylthio) Butanoic Acid to SulfurContaining Amino Acids in the Chicken Small Intestine. Poultry Science, 85: 1932-1938.

Melesse A, Dotamo E, Banerjee S, Berihun K, Beyan M. 2013. Studies on Carcass Traits, Nutrient Retention and Utilization of Koekoeck Chickens Fed Diets
Containing Different Protein Levels with Iso-Caloric Ration. Journal of Animal Science Advanced, 3(10): 532-543.

Miah MY, Chowdhury SD, Bhuiyan AKFH, Ali MS. 2014. Effect of different levels of dietary energy on growth performance of indigenous desi chicks reared in confinement up to target weight of 950 g. Livestock Research for Rural Development, $\quad \mathbf{2 6}, \quad$ Article \#124. Retrieved July 2, 2014, from http://www.lrrd.org/lrrd26/7/miah26124. html

Miah MY, Chowdhury SD, Bhuiyan AKFH. 2015. Effect of diets of different concentrations on indigenous growing chicks of Bangladesh in confinement. Asian Journal of Medicine and Biological Research, 1(1): 109-113.

Miah MY, Chowdhury SD, Bhuiyan AKFH. 2016. Effect of different dietary levels of energy on the growth performance and meat yield of indigenous chicken reared in confinement under the rural condition of Bangladesh. International Journal of Animal Research, 1(1): 53-60.

Mingh DV, Ogle B. 2005. Effect of Scavenging and Supplementation of Lysine and Methionine on the Feed Intake, Performance and Carcass Quality of Improved Dual-Purpose Growing Chickens. Tropical Animal Health and Production, 37: 573-587.

Mube KH. 2016. Besoins énergétiques et caractéristiques de production de la poule (Gallus gallus) Barrée villageoise. Thèse de Doctorat/PhD, Université de Dschang, Cameroun, P.150

Nasr J, Kheiri F. 2011 Effect of Different Lysine Levels on Arian Broiler Performances. Italian Journal of Animal Science, 10: 170-174.

Onu PN, Ayo-Enwerm MC, Ahaotu EO. 2010. Evaluation of Carcass Characteristics and Cost Effectiveness of Broiler Chicks Fed Synthetic Lysine and Methionine Supplemented Soya beanBased Diets. International Journal of Science and Nature, 1(1): 22-26. 
Plumstead PW, Romero-Sanchez H, Paton ND, Spears JW, Brake J. 2007. Effects of dietary metabolizable energy and protein on early growth responses of broilers to dietary lysine. Poultry Science, 86: 2639-2648.

Resnawati H, 1998. The nutritional requirements for native chickens. Bulletin of Animal Science, Supplement Edn, 522-527.

Saki AA, Mirzaaghatabar F, Zamani P, Aliarabi H, Hemati Matin HR. 2011. Energy Utilization by Chickens Fed Various Levels of Balanced Methionine. Global Veterinaria, 7(3): 276-282.

Sterling KG, Pesti GM, Bakalli RI. 2006. Performance of different broiler genotypes fed diets with varying levels of dietary crude protein and lysine. Poultry Science, 85:1045-1054.

Rezaei M, Moghaddam HN, Reza JP, Kermanshahi H. 2004. The effects of dietary protein and lysine levels on broiler performance, carcass characteristics and $\mathrm{N}$ excretion. International Journal of Poultry Science, 3: $148-152$.

Si J, Fritts CA, Waldroup PW, Burnham DJ. 2004. Effects of tryptophan to large neutral amino acid ratios and overall amino acid levels on utilization of diets low in crude protein by broilers. Journal of Apply Poultry Research, 13: 570-578. Stilborn HL, Mow JREE, Gous RM, Harrison MD. 1997. Effect of age on feather amino acids content in two broiler strain cross and sexes. Journal of Apply Poultry Research, 6: 205-209.

Swennen Q, Geraert PA, Mercier Y, Everaert N, Stinckens A. 2011. Effects of dietary protein content and 2-hydroxy-4methylthiobutanoic acid or DLmethionine supplementation on performance and oxidative status of broiler chickens. British Journal of Nutrition, 106: 1845-1854.

Trisiwi HF, Zuprizal, Supadmo D. 2005. Effect of the dietary protein level by essential amino ac id supplementation on carcass production of native chicken. Media Kedokteran Hewan, 21(3): 137143.

Yuan Y, Zhao X, Zhu Q, Li J, Yin H, Gilbert ER, Zhang Y, Liu Y, Wang Y, Diyan Li D, Yang Z, Shu G. 2015. Effects of dietary lysine levels on carcass performance and biochemical characteristics of Chinese local broilers. Italian Journal of Animal Science, 14:3. doi.org/10.4081/ijas.2015.3840 